https://doi.org/10.5719/aub-g/69.1/12

\title{
LECTURE DU RAPPORT COGNITIF AU PATRIMOINE DANS UNE PERSPECTIVE COMMUNICATIONNELLE : DECRYPTAGE DE LA DYNAMIQUE DES REPRESENTATIONS CULTURELLES ET LEUR RECEPTION. CAS DU CENTRE HISTORIQUE D'ALGER
}

MANSOURI LAMIA ${ }^{1}$, BOUARROUDJ RADIA ${ }^{2}$, DEBACHE SAMIRA ${ }^{3}$

\author{
READING OF THE COGNITIVE REPORT TO HERITAGE FROM A COMMUNICATION \\ PERSPECTIVE: DECRYPTING THE DYNAMICS OF CULTURAL \\ REPRESENTATIONS AND THEIR RECEPTION. \\ CASE OF THE HISTORICAL CENTER OF ALGIERS.
}

Résumé

Le patrimoine bâti est une composante essentielle du lieu. Il est indissociable d'un ensemble physique et social qui lui permet de signifier et auquel il donne sens. L'acceptation de ces termes inscrit le patrimoine dans la construction collective d'un bien commun basée sur le regard qualifiant porté par la société du présent sur le legs de celles qui l'ont précédée. Le réinvestissement du passé par le présent met en exergue l'importance de concevoir la construction symbolique du patrimoine plutôt que l'objet constitué. Dès lors le patrimoine n'est plus appréhendé en fonction de sa matérialité mais de son opérativité sociale.

L'intellection de la notion ainsi posée impose l'impératif d'envisager la dimension appropriationnelle et les dynamiques d'interaction d'un groupe social avec son héritage.

L'hypothèse avancée est que cette appropriation est indexée à une construction culturelle d'une valeur patrimoniale au fait de la médiation.

Afin d'élucider ces propos, le choix s'est porté sur le site de la casbah d'Alger, centre historique de la ville. Au travers de cet espace urbain, nous tenterons d'évaluer et

$1 \quad$ Université d'Alger 1. lamianagati@yahoo.fr

2 Université de Constantine III, dadoo_162002@yahoo.fr

3 Université de Constantine III, samira.debache@univconstantine 3.dz 
de comprendre les termes du rapport d'appropriation cognitive que développent les pratiquants du site avec le patrimoine bâti en évaluant l'impact de la médiation dans cette configuration particulière.

Mots clés: Appropriation cognitive - médiation patrimoniale - casbah d'Alger-approche qualitative.

\section{Introduction}

Le patrimoine connait ces dernières années une transformation profonde qui implique le passage d'une perspective historique vers une approche plus sociologique comme le développent Boudin (1984), Lamy (1996), Noppen et Morisset (2004) et, Amougou (2004).

Ces auteurs consacrent l'idée selon laquelle la valeur patrimoniale n'est plus restrictive aux qualités intrinsèques d'un objet, mais relative à une perception et une subjectivité collective dépendantes des conditions sociales et historiques propres à un contexte. L'acceptation de ces termes inscrit le patrimoine non seulement entre la responsabilité de la préservation d'un legs mais aussi dans la construction collective d'un bien commun, mettant en exergue sa fonction sociale définie par le degré d'attachement d'une société à son héritage. Ce recentrage impose l'appropriation comme un concept majeur dans l'équation patrimoniale.

L'hypothèse retenue est que cette dimension appropriationelle exige la mise en relation du patrimoine avec des sujets récepteurs par toutes formes d'actions et de démarches menées dans la perspective de son affirmation en tant que symbole représentatif du passé.

D'objet exemplaire, le patrimoine devient un médiateur au sens communicationnel entre deux univers : le monde de référence du patrimoine et celui du public. Nous supposons de ce fait que la construction communicationnelle du rapport au passé est constitutive de l'acte d'appropriation du patrimoine.

Cette posture épistémologique oriente le choix méthodologique vers une approche qualitative concrétisée au travers de l'enquête par entretien. Sa configuration comme dispositif de narration est apte à repérer dans le discours l'ensemble des significations à l'œuvre dans la compréhension du rapport cognitif des pratiquants du centre historique d'Alger au patrimoine bâti du site en évaluant de l'impact de la médiation dans cette configuration particulière. 
LECTURE DU RAPPORT COGNITIF AU PATRIMOINE

DANS UNE PERSPECTIVE COMMUNICATIONNELLE : ...

\section{Appropriation : polysémie d'un concept}

Il existe une intensité d'échanges autour de ce concept qui induit à une sorte de polysémie de sens sur les définitions à fournir.

Selon Perla Serfaty-Garzon (2003 : 27), la notion d'appropriation véhicule deux idées dominantes. D'une part, celle d'une adaptation de quelque chose à un usage défini ou à une destination précise; d'autre part, celle, qui découle de la première action visant à rendre propre quelque chose. En psychologie environnementale, l'expérience du lieu repose sur les interactions entre l'individu et l'environnement. Pour Gustave-Nicolas Fischer(1992: 91), elle peut être définie comme un « système d'emprise sur les lieux.» Il s'agit d'un pouvoir à la fois physique et psychologique sur un espace individuel ou collectif. Un moyen de matérialiser une partie de son univers mental dans l'espace environnant afin de le familiariser et de s'y sentir en sécurité.

Dans cette logique le patrimoine bâti qui connait des extensions impliquant son apparenté à un espace ou un ensemble d'espaces représentera «l'archétype du bien approprié. » (Godard 1990 : pp. 215-241)

\section{I.1. L'appropriation cognitive}

L'appropriation cognitive fait partie des modalités d'appropriation à dominante idéelle qui sont "inséparables d'intentions, de perceptions et représentations, et même de constructions imaginaires ou idéologiques... [qui supposent] une pratique concrète, régulière et démonstrative de l'espace. " (Ripoll et Veschambre 2005 : 7-15.)

Dans le domaine du patrimoine, elle matérialise le développement d'une connaissance approfondie théorique et pratique par apport à l'objet patrimonial permettant d'en user de façon pertinente ou stratégique (Ripoll et Veschambre.V 2006 : 295-304).

Le premier indicateur de cette appropriation sera concrètement accompli au travers du langage par un acte de désignation. 


\section{La médiation patrimoniale : une interface communicationnelle.}

Dans son sens littéral, la notion de médiation met l'accent sur le lien, la conciliation. Ses «fonctions sont d'accompagner, de réguler ou de négocier grâce à un tiers, une fracture entre des mondes qui seraient séparés, ou encore de permettre la communication entre ces mondes. »(Gellereau, 2005 : 27-42.)

Cette notion aborde le fait qu'un sujet inscrit dans un processus de médiation est conduit à explorer et à confronter d'autres points de vue à son propre regard (Darra $2004: 61-85$ ) dans une dialectique constante entre la rationalité et la sensibilité du sujet. Sous cet angle, le sens ne serait plus immanent aux objets mais se construit par des sujets grâce à des langages et des dispositifs qui permettent l'émergence d'un monde composé d'interprétations diverses.

La médiation culturelle s'inscrit dans mouvement qui attache une importance nouvelle à la question du rôle des publics dans l'équation culturelle. La médiation du patrimoine s'inscrit pleinement dans ce schéma et se situe dans la dynamique des représentations culturelles et leur réception. Elle engage des dispositifs pour transmettre et communiquer des savoirs et des valeurs en construisant "une interface entre deux univers étrangers l'un à l'autre (celui du public et celui, disons, de l'objet culturel) dans le but précisément de permettre une appropriation du second par le premier. » (Davallon 2004 : 37-58.)

L'objectif étant de produire une signification symbolique reliée aux différentes valeurs du patrimoine afin d'opérer l'appropriation de ce dernier par une communauté dont il représente l'héritage. Cette nouvelle réalité $\mathrm{du}$ patrimoine force l'articulation entre sa dimension référentielle et sa dimension communicationnelle.

La première étant basée sur les attributs traditionnels du patrimoine (authenticité et véridicité). Elle est identifiable à travers des signes qui renvoient aux qualités intrinsèques de l'objet mais aussi à son monde de référence (dates des vestiges, références historiques...etc.). La deuxième s'articule autour de la notion « d'expérience » qui vise une construction de sens et sera plutôt orientée vers une manière d'appréhender l'objet patrimonial en usant de dispositifs médiatique (signalétiques scénographie décors, reconstitution, mise en scène, film images, spectacle vivant ... etc.) 


\section{Présentation du contexte d'étude}

Alger doit sa naissance à sa situation géographique, la topographie de son site et surtout à son port, qui depuis l'Antiquité lui vaut un attrait particulier qui a constitué le premier vecteur de son urbanisation.

Les Phéniciens y établirent un emportium, un de ces comptoirs qui jalonnaient leurs routes maritimes (Ichoubédene $1997: 23$ ).

Après une période de flottement où le comptoir fit partie du royaume de Maurétanie indépendant et gouverné par les rois berbères, il fut annexé en l'an 40 à l'empire romain. Vers le $X^{e}$ siècle la tribu berbère des Sanhadja se fixe sur l'emplacement des romains. Le Prince Bologhine Ibn Ziri fonda vers l'an 950 de notre ère le premier noyau de la médina nommée El Djazair (Djilani 2005 : p. 16). La prise du pouvoir par les Ottomans au XVIème siècle, contribuera à l'essor extraordinaire de la ville qui accède au sommet de la hiérarchie urbaine du pays. De Bourgade, Alger devient « l'espace central d'un pouvoir politique rayonnant sur le Maghreb» (Ichoubédene 1997 :19). En 1830, la rupture historique que représente la colonisation française métamorphose complètement la ville par ce qu'elle engendre comme destruction et substitution des ordres urbains. Marginalisée pour d'autres centralités, la médina désormais nommée la Casbah va glisser du statut d'un centre de pouvoir vers celui d'un quartier marginalisé de la ville.

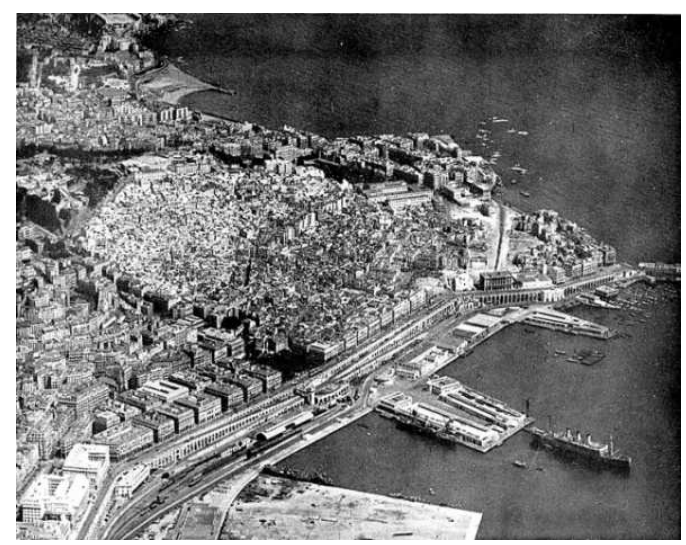

Fig. 1. Vue aérienne de la casbah d'Alger. Source : /www.algerie-ancienne.com 
A la période post indépendance les bouleversements liés au nouveau rôle d'Alger en tant que capitale d'une nation indépendante donne lieu à une radicale réutilisation de l'espace urbain.

Cette situation eue des répercussions sur le centre historique qui se sont traduits dans les faits par une déstabilisation de la structure sociale et physique engendrant une marginalisation spatiale, économique et sociale. Le site fut classé patrimoine mondial par l'Unesco en1992 et instauré en secteur sauvegardé en mai 2005.

\section{Une méthode qualitative opérante in situ}

Le questionnement qui porte sur la compréhension du rapport d'appropriation cognitive que développent les pratiquants du centre historique au patrimoine bâti doit "se construire intellectuellement à partir de données brutes recueillies et à travers une mise en concept ainsi qu'à travers des efforts intellectuels de compréhension destinés à faire apparaitre du senspour-des-acteurs en-situation. » (Mucchielli 1996)

La méthode qualitative apparait de ce fait pertinente pour la réalisation de cet objectif.Elle permet de mettre l'accent sur les acteurs afin d'avoir accès à leur interprétation d'une réalité sociale par l'intermédiaire de la compréhension et la signification qu'ils ont du monde qui les entoure et dans lequel ils se sont engagés.

Deux axes articulent l'investigation :

- La Compréhension et l'évaluation du degré et des formes d'appropriation cognitive au patrimoine bâti développées par les enquêtés.

- La mise en rapport de cette appropriation avec les stratégies communicationnelles (traduite par les différents modes et dispositifs de médiation) existantes sur site.

Ce qui revient à évaluer l'impact de la médiation dans la construction d'un lien avec le passé au travers des objets patrimoniaux.

L'entretien semi-directif est mobilisé pour la réalisation de cet objectif. Cet outil méthodologique de narration représente une technique de communication et d'interaction humaine ayant la capacité de par son 
caractère immersif et la parole performative du discours à révéler les dimensions et les logiques explicatives du phénomène social étudié.

\section{IV.1. Le corpus d'étude}

L'objectif de définir un rapport d'appropriation cognitive porte sur la globalité du centre historique qui est d'ailleurs classé comme patrimoine universel et comme secteur sauvegardé. Cependant la faisabilité de l'enquête, a exigé de travailler sur les éléments saillants. Le corpus d'étude comportera une liste de monuments classés parmi les plus emblématiques du site. Dans sa constitution il intègre plusieurs typologies et aura pour limite chronologique la période ottomane ${ }^{4}$.

Il se composera de :

- La mosquée sidi Ramdane située dans la partie haute de la casbah date de l'époque des dynasties berbères médiévales. (Devoulx 1870 : 226) (fig. 2)

- La mosquée Djamaa el kebir, bâtie par le souverain Almoravide Youssef Ibn Tachfin en 1097. (Bourouiba 1983 : 62)

- La mosquée Ketchaoua : des hypothèses font remonter sa construction à une période antérieure au XVIe siècle. Elle sera reconstruite au XVIII siècle par le dey Hassan pacha (Djermoune \& Oubouzar 2013 : 249). Sous l'occupation française, elle fut massivement remaniée et affectée au culte catholique pour reprendre son usage initial à l'indépendance de l'Algérie. (fig. 3)

- Le mausolée Sidi Abderrahmane El-Thaalibi, saint patron d'Alger édifié en1611, il sera remanié en 1696, sous le dey El-Hadj-Ahmed puis transformé à nouveau en 1729 à l'époque d'Abdi-Pacha. (fig. 4)

- Le palais Dar Khedaoudj construit en 1570 dans la partie basse de la casbah (CNRA) ${ }^{5}$. Aujourd'hui il abrite le musée des arts et traditions populaires.

4 Le patrimoine colonial véhicule des valeurs historiques et des valeurs d'usages qui nécessitent une lecture spécifique du rapport d'appropriation.

5 Centre nationale des recherches archeologiques. 
- Le palais Aziza fondée entre 1552 et 1556 (CNRA), fut la résidence du Dey pour devenir 1er siège de la régence à l'époque ottomane. Il est actuellement le siège de l'office national de la gestion des biens culturels. (fig. 5)

- Le palais Hassan pacha fût construit en 1791 par le Dey d'Alger Hassen El-Kheznadji (Golvin2003 : 62). Il subira plusieurs modifications à l'epoque coloniale. Il est aujourd'hui une administration affiliée au ministère des affaires religieuses. (fig. 7)

- Le palais des Rais fut édifié aux 16 siècles par Mustapha Pacha, (CNRA). Le bâtiment est la dernière survivance du rapport de la médina avec la mer. Il abrite actuellement le centre des arts et de la culture. (fig. 6)

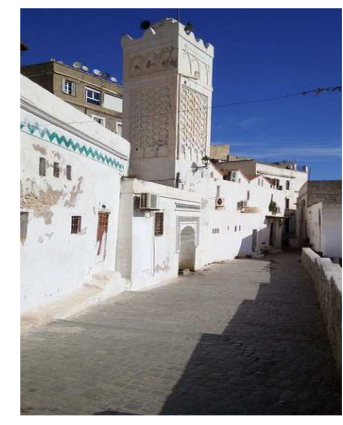

Fig. 2. La mosquée Sidi Ramadane (Source : Auteur, 2018)

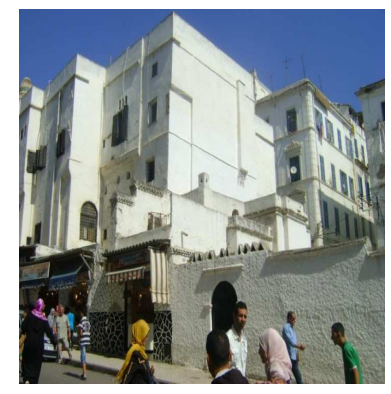

Fig. 5. Palais Aziza (Source : Auteur, 2019)

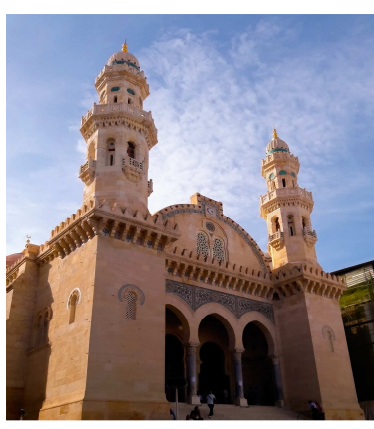

Fig. 3. La mosquée Ketchaoua (Source : Auteur, 2018)

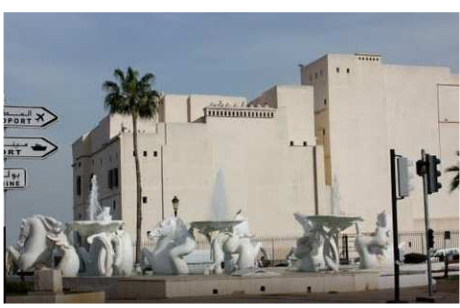

Fig. 6. Palais des Rais (Source: cnra.dz)

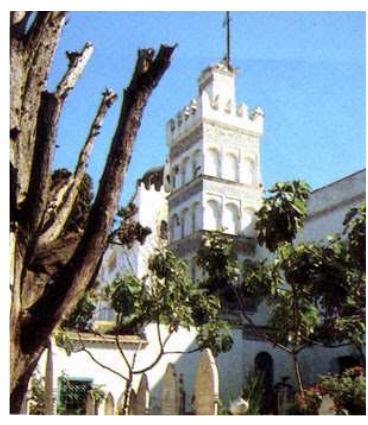

Fig. 4. Mausolée Sidi Abderrahmane (Source : cnra.dz)

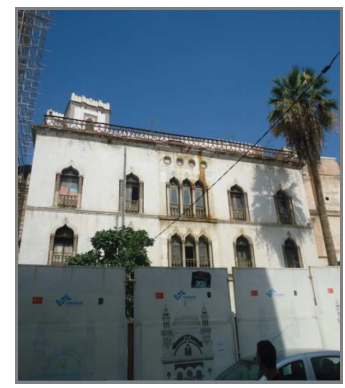

Fig. 7. Palais Hassan pacha (Source : Auteur, 2018) 


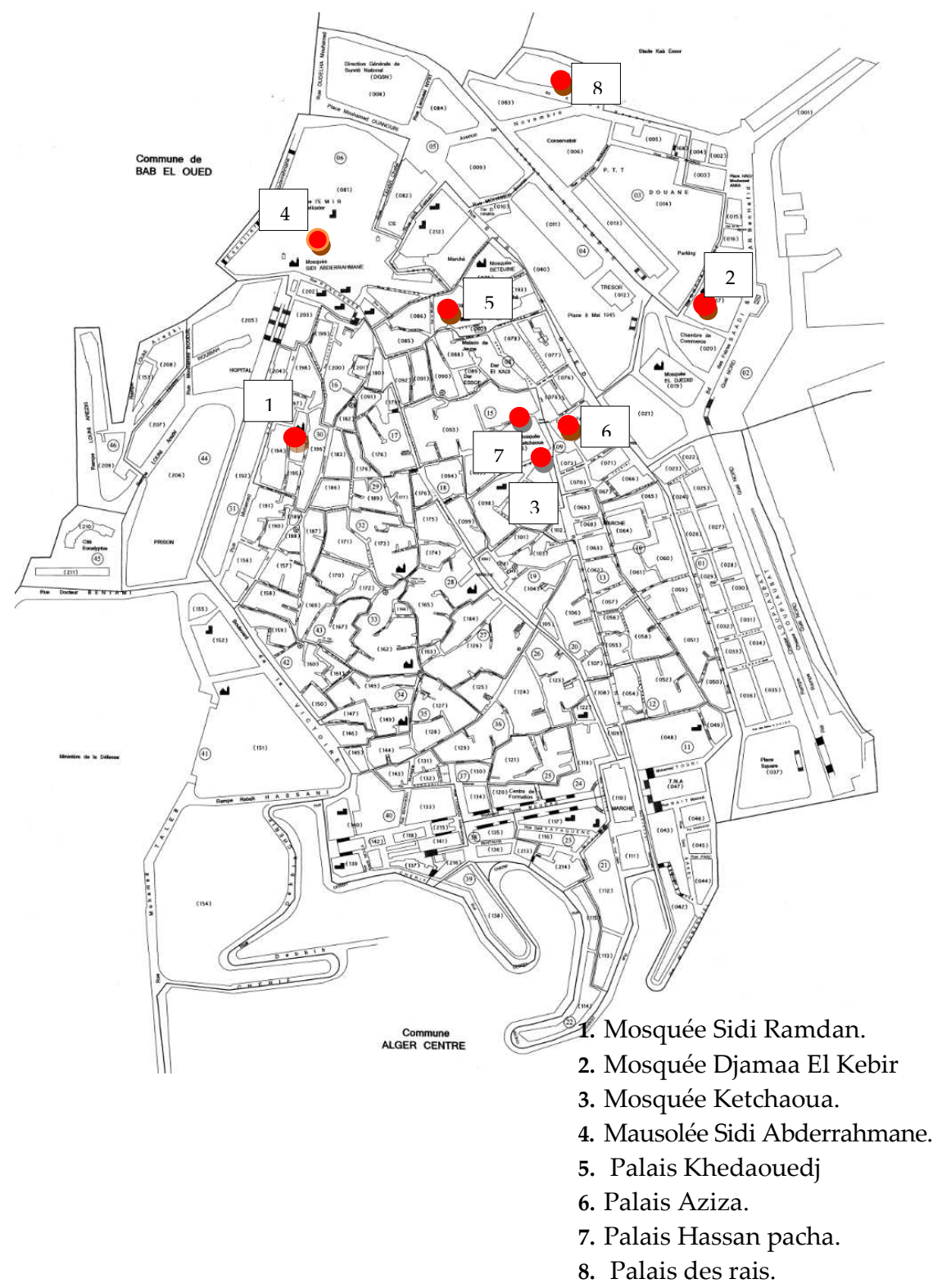

Fig. 8. Emplacement des éléments du corpus (Source: Auteur, 2019) 


\section{IV.2. L'échantillon des enquêtés : critères et modes de représentativité}

L'échantillon de l'enquête repose sur le principe d'une représentativité théorique. Il sera apparenté à un échantillonnage typique où tous les éléments choisis sont des modèles représentatifs de la population ciblée.

L'objectif étant de mettre en évidence une grande diversité de situations et d'établir une typologie la plus proche de l'exhaustivité des configurations possibles dans le but de reconstruire la composition sociologique du contexte d'étude.

Le critère de représentativité qui guide la sélection des enquêtés est celui de la pratique du centre historique.

Pratiquer les lieux ne revient pas au simple fait de la fréquentation, il s'agit d'une notion qui insiste sur le faire et sur les actes.

«Chaque pratique exprime un certain habiter à l'échelle de l'individu» (Stock 2003 : 213-229)

"Un même lieu n'a pas le même sens selon les pratiques qui s'y déploient. Chaque pratique met donc en jeu l'espace d'une façon nouvelle: c'est l'enseignement que l'on peut tirer d'une lecture pragmatique de l'espace ». (Stock $2007 ; 103-125)$

Mais la pratique d'un un lieu n'induit pas seulement faire expérience de sa matérialité, mais aussi en donner une signification particulière et symbolique. Cette dernière est construite par le travail de traduction des valeurs existantes que par l'injection de nouvelles valeurs véhiculées par le vécu.

Ce concept renvoie à l'action que l'individu peut avoir sur un espace et la signification qui lui octroie en fonction de son propre vécu et des particularités du site.

Trois typologies ont été intégrées à l'échantillon pour répondre à toutes les configurations possibles :

- Les pratiquants qui résident sur le site de façon permanente (locataires et propriétaires.) (6personnes)

- Les pratiquants qui travaillent sur le site. (5 personnes) 
- Les personnes qui pratiquent le site de façon occasionnelle (commerces, services, ... etc.) (9 personnes)

L'échantillon exclut des usagers spécifiques que sont les touristes. Ils sont présents sur le site d'une manière beaucoup trop sporadique pour être relevée et intégrée à l'enquête.

Le critère sociodémographique fut mobilisé afin d'optimiser la diversité de l'échantillon et d'assurer la plus grande hétérogénéité de la population interrogée :

- L'âge : toutes les personnes interviewées lors des entretiens étaient majeures (les enfants sont une catégorie qui n'est pas prise en compte).

- Le genre : une parité a été recherchée afin d'aboutir à un effectif équilibré homme/femme. (11 femmes/9hommes)

- Le niveau d'instruction et les catégories socioprofessionnelles : cette information a servi à veiller à ne pas restreindre le discours à une catégorie spécifique de la société. (niveau primaire /moyen $40 \%$ - 35\%secondaire - universitaire $25 \%$ )

\section{IV.3. Grille d'entretien : un cadre souple et évolutif}

La capacité à s'approprier le patrimoine par les pratiquants du centre historique est subordonnée à une compétence cognitive qui est définie comme la manipulation du savoir dans le discours.

Une grille d'entretien permettra la mise en exergue de cette dernière par un système organisé de thèmes qui structure le questionnement mais ne dirige pas le discours traçant un cadre souple et évolutif aux entretiens.

Un volet introductif servira à cerner les caractéristiques personnelles de chaque enquêté (âge, sexe, niveau scolaire, profession) ainsi que sa catégorie d'appartenance à la typologie de notre échantillon.

La deuxième partie sera scandée par deux axes de d'investigation :

Le premier aura comme objectif l'évaluation du capital informatif de l'enquêté sur le patrimoine bâti du site (capacité à identifier des éléments du patrimoine bâti et aptitude à développer des connaissances sur les éléments du corpus.) 
La compétence cognitive se décline aussi selon un ordre spatial (capacité à s'orienter, à localiser un édifice, et pouvoir y accéder.)

Cette compétence a été étudié dans une relation de causalité avec l'existence de stratégies communicationnelles consacrées au site (médiation in situ et ex situ) afin d'établir l'impact de la médiation sur les configurations d'appropriation cognitive au patrimoine bâti.

\section{IV.4. Protocole d'analyse du corpus}

Le protocole d'analyse repose sur la thématisation continue une des méthodes d'analyse de contenu. Elle consiste à décomposer le corpus de verbatim constitué par les entretiens en des thèmes représentatifs et synthétiques du contenu analysé (Mucchielli 2008 : 62).

Le premier palier d'analyse a consisté en un repérage de ces unités thématiques qui composent l'univers discursif des enquêtés. Ces dernières représentent des partitions porteuses de sens, identifiables en fonction de la problématique et des hypothèses de recherche.

Elles permettent de produire une reformulation du discours sous une forme condensée et formelle constituant ainsi le cadre de l'analyse. Leur présence ou leur fréquence d'apparition signifie quelque chose pour l'objectif analytique choisi. Trois logiques narratives énoncent les domaines au travers desquels furent relevées et analysées les postures d'appropriation cognitive au patrimoine. : L'identification, l'orientation et la connaissance.

\section{L'appropriation cognitive au prisme de la médiation : Efficience et Carence des stratégies communicationnelles}

\section{V.1. Identifier, nommer : la toponymie comme facteur d'appropriation}

La dimension cognitive se traduit dans une première acception par la capacité à reconnaitre et à nommer des éléments du patrimoine bâti.

"La ville a une toponymie propre qui est un facteur d'appropriation" (Feinanguai 2004 : 244-255.) 
LECTURE DU RAPPORT COGNITIF AU PATRIMOINE

DANS UNE PERSPECTIVE COMMUNICATIONNELLE : ...

Cette désignation des lieux, est alors révélatrice de l'appartenance à un groupe et l'adhésion à une représentation spécifique de l'espace.

Dans cet ordre d'idées, le premier palier dans l'évaluation de la compétence cognitive dans le discours des enquêtés revenait à estimer l'aptitude de ces derniers à identifier et à nommer dans leur parcours habituels du site des éléments saillants du patrimoine bâtíi

La grille d'entretien mentionne le vocable d'édifice remarquable du point de vue de l'importance des ses dimensions, de ses qualités esthétiques (éléments décoratifs ou architectoniques) afin de ne pas spécifier aucun terme technique inaccessible aux enquêtes (édifices classé, monuments...etc) laissant à ces derniers la liberté de designer dans le tissu urbain les éléments qu'ils estiment importants.

Dans l'analyse du verbatim, il ressort une constante : la capacité des résidents à définir la toponymie des édifices. Le fait d'habiter le site, le transforme en un espace vécu, un espace connu ce qui permet la constitution de repères cognitifs.

«C'est évident que je peux nommer ce que je perçois... c'est mon quartier!» (Abdelkader 67 ans résident à la casbah.)

"Oui bien sur j'arrive à identifier tous les édifices importants que je rencontre, les mosquées, les palais, et même les fontaines et les rues... Je suis de la Casbah » (Salah 27ans résident la casbah)

Pour le reste des pratiquants cette capacité est très variable selon les profils. Les enquêtés qui travaillent sur le site peuvent reconnaitre et nommer avec plus ou moins d'aisance les édifices patrimoniaux qui ponctuent leurs parcours.

Cette aptitude s'exprimera d'une manière irrégulière selon l'expérience individuelle de chacun. En se référant aux propos des enquêtés, nous la reportons à leur pratique récurrente du site et leur proximité avec les habitants de la casbah.

6 Le choix du parcours habituel aura comme objectif de souligner une pratique récurrente de l'espace ce qui permet une proximité spatiale avec le lieu. 
"Je connais pas mal de choses à la casbah... Je discute beaucoup avec les gens du quartier, c'est un grand plaisir pour moi! Ces personnes sont la mémoire vivante de la casbah et puis comme je vous l'ai dit je suis ici depuis longtemps!» (Aziz 55 ans commerçant)

Les pratiquants occasionnels du site affichent dans leur propos une difficulté visible et variable à nommer les édifices patrimoniaux présents dans leurs parcours bien qu'ils énoncent clairement discerner dans le tissu urbain des éléments qui par leur caractère esthétique et ont attisé leur curiosité.

"Je fais souvent des courses dans la basse casbah...je connais la mosquée ketchaoua comme tout le monde et la mosquée Djemaa ejdid... pour le reste il est vrai que des grandes bâtisses bordent la moquée ketchoua,... je crois qu'elles sont turques..., mais je n'ai jamais fais la démarche de me renseigner sur leurs noms.» (khaoula 33 ans pratiquante occasionnelle du site)

«...pour ma part quand un édifice m'interpelle je demande aux gens du quartier de me donner le nom... c'est la seule manière à mon sens d'avoir la réponse..ou bien aller sur le net mais c'est plus compliqué car on a pas le nom pour lancer la recherche » (Karim 27 ans pratiquant occasionnel du site.)

Si nous devions souligner les dénominateurs communs aux entretiens, les édifices cultuels se révèlent comme les plus cités par les enquêtés. Leur monumentalité, leur qualité esthétique et leur valeur d'usage ne manquent pas, $d$ 'insuffler aux personnes interrogées une charge identitaire et spirituelle, faisant d'eux des repères cognitifs importants du site.

Le palais des Rais émerge aussi dans le discours de par sa position dominante et excentrée par apport à la médina mais aussi par sa présence récurrente dans les médias.

Cette défaillance cognitive, il est important de le souligner, est reportée par les enquêtés, à l'absence d'éléments informatifs sur site. 


\section{V.2. Orientation / localisation : pour une intelligibilité des lieux}

La capacité à s'orienter dans l'espace se réfère à une habileté mentale c'est-à-dire la capacité à appréhender, identifier à partir de ses attributs majeurs les caractéristiques d'un environnement.

Cette image se forme par une véritable reconstruction interne de l'espace tel que l'individu le perçoit selon des modalités sensorielles multiples. C'est un processus cognitif où l'usager a recours à des signes et à des informations puisés dans l'espace et qu'il juge pertinents à ses besoins.

Nous pouvons de ce fait avancer que l'orientation spatiale émerge grâce à l'élaboration d'une signification qui se construit à partir de la compréhension subjective d'un lieu.

Cette aptitude à s'orienter est reportée par Passini (1996 : 319-331) à un facteur d'appréciation et d'appropriation de l'environnement bâti. Dans le sens où il l'entend, Passini accorde à ce concept d'appropriation une valeur instrumentale et cognitive car il implique la capacité d'utiliser efficacement l'espace.

Cet environnement qui dans notre cas est un espace urbain se présente comme relais et émetteur d'informations. Parmi celles-ci, Passini et Shiels (1987) en signalent deux catégories dont l'assimilation permet à l'usager de se représenter l'organisation de son milieu.

1. Les méta-informations, c'est à-dire les informations qui permettent de comprendre la structure de l'environnement. Elles renvoient aussi bien à l'environnement qu'à l'espace architectural.

2. Les micros informations qui en permettent l'usage comme les outils signalétiques (les flèches signalétiques, les tableaux d'orientation, éléments de balisage et de jalonnement... etc.)

La transposition de ces données au contexte d'étude converge vers l'évaluation de la capacité des enquêtés à localiser les édifices patrimoniaux importants (éléments du corpus) qui se présentent aussi comme des éléments de repères au niveau du centre historique. Cette compétence traduit une maitrise probante de l'espace.

$\mathrm{Au}$ travers de notre enquête nous avons constaté que cette représentation spatiale cognitive se construit avec l'expérience et la pratique. 
Cet apprentissage se base sur le rapport stimulus-réaction par apport aux informations émises par l'environnement bâti, ce qui oriente la perception et anticipe la décision.

Le profil des résidents obéit à ce schéma. Nous relevons dans leur propos une bonne intelligibilité de l'espace qui transparait dans leur capacité à se représenter les caractéristiques de leur environnement.

Ils arrivent ainsi à localiser les édifices patrimoniaux du corpus en se référant aux méta-informations du site (les voies, les caractéristiques architecturales... etc.)

"La mosquée Sidi Ramdane, est une petite mosquée qui n'est pas loin d'ici, dans la haute casbah [...] La mosquée Ketchaoua est dans la basse Casbah dans le quartier Souk el Djemaa à coté de Dar Aziza... Dar Hassan Pacha qui est le siège des affaires religieuses est en face de Dar Aziza. Djamaa el kebir est sur le boulevard du $1^{\text {er }}$ Novembre [...] enlongeant la rue on trouve le palais des Rais. Le mausolée Sidi Abderrahmane est dans la haute Casbah pas loin de la rue Marengo... » (Abdelkader 67 ans résident à la casbah)

Cette capacité est aussi présente chez les travailleurs sur site mais s'exprimera d'une manière différente : les enquêtés seront à même de localier avec précision les édifices qui se situent dans une zone proche de leur lieu de travail.

"La mosquée Sidi Ramdane se trouve dans la haute Casbah, pas loin de l'hôpital à une ruelle d'intervalle. Pour le mausolée Sidi Abderrahmane il faut descendre encore des ruelles, il est facile à repérer, vous pouvez demander, tout le monde le connait ; la mosquée Ketchaoua, est dans la basse Casbah. La grande mosquée est aussi dans la basse Casbah mais en contrebas, plutôt vers la mer, le palis des Rais aussi. Les palais Dar Khdaouedj, j'en entends parler mais je ne saurais pas le situer. Le palais Hassan pacha je n'en ai jamais entendu parler.» (Souhila 42 ans, infirmiére travaille sur site.)

Par opposition, nous relèverons dans la diversité des réponses et des expériences que les pratiquants occasionnels entreprennent avec le site, 
une difficulté à s'orienter mais aussi à dresser une localisation des éléments du corpus.

Cette inaptitude traduit leur incapacité à établir des repères qui leur permettent de se représenter cognitivement le centre historique. $\mathrm{Ce}$ manque de repères cognitifs et spatiaux n'est pas comblé par les médiations in situ (micros informations).

"Sur tout les édifices que vous venez de citer, je peux vous localiser que trois, la mosquée ketchaoua,la mosquée djamaa el Kebiret lepalais des Rais pour le reste je ne sais pas. » (Radia 46 ans pratiquante occasionnelle du site.)

"C'est très difficile de s'orienter. Pour trouver le musée du palais khedaouedj nous avons demandé deux fois notre chemin... Ce n'est pas normal. Il faut un minimum, de renseignements, au moins pour retrouver les musées ou les monuments, pour que les gens puissent y venir. » (Amina 32 ans pratiquante occasionnelle du site.)

Dans ce contexte la signalétique interprétative et directionnelle aurait pu contribuer à l'intelligibilité des lieux.

La première en identifiant et nommant les lieux et les édifices patrimoniaux, apporte de l'information là où elle manque, offrant une vision plus explicite du contexte.

La deuxième aura à la fois la vocation et d'éviter le sentiment de désorientation et diriger le pratiquants occasionnels vers les éléments considérés comme importants de par leur valeur historique ou architecturale.

Elle améliore de ce fait la compétence de pratique chez l'enquêté et sa capacité à anticiper et à choisir ses parcours dans le centre historique.

Cette stimulation de la capacité à prévoir optimise «le travail de reconnaissance et d'appropriation d'un contenu culturel. » Jacobi Daniel, Jeanneret Yves $2013: 47-72)$

\section{V.3. Connaissances et compréhension : l'accès au savoir}

Les mécanismes cognitifs sont analysés dans le discours au travers de l'aptitude des enquêtés à développer des connaissances sur le centre 
historique (évaluation du capital informatif sur le centre historique dans sa globalité mais aussi sur les éléments patrimoniaux du corpus).

Un parallèle est fait avec les stratégies communicationnelles et les différentes actions de médiation opérantes sur site afin d'évaluer leurs impact sur les configurations d'appropriation.

La première constante relevée dans les entretiens est une conscience partagée par tous les enquêtés du statut de la Casbah comme patrimoine universel.

Cet état de fait est ramené par ces derniers à une présence récurrente de cette information dans les medias au point d'être automatiquement associée au site.

"Oui je le sais, cette information est présente partout, à la télévision, à la radio. Dés qu'on parle de la Casbah on dit qu'elle est mondialement classée. Moi je ne vois pas sincèrement l'avantage qu'a apporté ce classement. " (Salah 27 ans résident la casbah.)

Le statut de secteur sauvegardé pour sa part, est connu principalement par les résidents en rapport aux différentes restrictions et législation concernant le cadre bâti.

"Oui je sais... d'ailleurs on a plus le droit de modifier quoi que ce soit dans la maison sans en revenir aux services concernés » (Abdelkader 67 ans résident à la casbah.)

Le deuxième constat que nous révèle l'analyse du discours est les configurations différentes dans l'acquis cognitif des enquêtés :

Les résidents présentent une bonne connaissance des éléments patrimoniaux du corpus, cependant certains édifices sont reconnus par leur fonction qui vient supplanter leur valeur historique (comme le palais Hassan Pacha qui est reconnu par sa fonction de Siège des affaires religieuses).

Le capital informatif développé par les résidents est appréciable (plus présents chez les personnes âgées.) Ce savoir qui ne relève pas toujours d'une véracité historique est essentiellement relié à une mémoire des lieux et à une transmission intergénérationnelle de la part des anciens qui dans ce cas peuvent être considérés comme des médiateurs du site. 
LECTURE DU RAPPORT COGNITIF AU PATRIMOINE

DANS UNE PERSPECTIVE COMMUNICATIONNELLE : ...

Ces derniers agissent sur la réception active du sujet social et sa capacité non seulement à acquérir les connaissances transmises, mais lui permettent de les relier à leurs propres expériences pour ainsi produire chez lui du sens et de la signification.

"Oh tout d'abord le récit des anciens, mes parents, mes grands parents. Il y'a tellement d'histoire et de légendes autour de ce quartier. » (Kamir 60 ans résidente à la casbah.)

"Il y'a des choses que j'ai toujours su, sans savoir exactement qui me les a apprises, forcement ça doit être ma famille et particulièrement mon père. Pour le reste j'ai constitué mon propre savoir, principalement au travers des livres. » (Radia 30 ans résidente à la casbah.)

Ce rôle de médiateur attribué aux anciens est aussi repris par les travailleurs sur site, qui considèrent pour leur part que ces derniers sont un vecteur important de la transmission de savoir sur la Casbah et qu'ils devraient être intégrés à tout projet visant la valorisation du site.

«La meilleure manière façon de connaitre la Casbah c'est de la visiter et pour bien la visiter il faut le faire avec les gens du quartier,et surtout les anciens c'est un capital à exploiter au travers d'associations par exemple. » (Lyes 35 ans travaille sur site.)

Pour les deux autres profils (travailleurs et pratiquants occasionnels), notre constat par apport au premier palier d'évaluation, celui de la reconnaissance (affirmer connaitre des éléments du corpus), nous a permis d' avancer les résultats suivants:

- Le niveau de connaissance des travailleurs sur site est plus élevé que celui des pratiquants occasionnels.

- Les édifices cultuels sont les plus cités par les enquêtés et émergent comme des repères cognitifs du site.

- Les édifices patrimoniaux ouverts à la visite sont relativement plus cités (palais des Rais, palais khdaouedj) par les pratiquants 
occasionnels que ceux affectés aux administrations, ce qui justifié par leur accessibilité et leur présence dans les médias. ${ }^{7}$

En termes d'information, il ressort des entretiens une connaissance, relativement sommaire peu mobilisée ou alors de manière erronée.

Cet état de fait est perceptible pour les deux catégories d'enquêtes dans la diversité des niveaux d'instruction.

Ces derniers avancent des informations inexactes ignorant par exemple les limites de la Casbah, ses phases d'évolution, ou peuvent associer des édifices à des époques largement ultérieures à leur périodes d'édification. (A titre d'exemple des enquêtés présentent les deux mosquées Sidi Ramdane et Djamaa El Kebir comme des constructions ottomanes.)

Cette méconnaissance est reportée par les enquêtés au sentiment de ne pas avoir la culture ad hoc concernant le centre historique et que l'absence d'éléments informatifs sur site entrave la constitution d'un capital cognitif.

« il n'ya pas de renseignements sur l'histoire et la valeur de la Casbah, on vient, on repart plusieurs fois en se contentant de contempler avec les yeux c'est tout. » (Hamid 35 ans pratiquant occasionnel du site.)

"C'est dommage parce que c'est une ville qui recèle beaucoup d'éléments de patrimoine et très peu de gens ont la culture pour les connaitre ou les comprendre. L'information est nécessaire pour tous les autres, pour qu'ils puissent y accéder. » (Imen 45 ans pratiquante occasionnelle du site.)

- En exprimant leurs besoins, les enquêtés considèrent que la signalétique est le dispositif informatif le plus opérant sur site pour la transmission de connaissances sur le centre historique d'Alger. La visite guidée est aussi citée comme un canal privilégié à cet objectif, particulièrement lorsqu'elle est portée pas les personnes originaires du quartier.

- Ex situ, l'internet est envisagé comme une voie importante pour diffuser et relayer l'information sur le centre historique.

7 Le croisement des réponses des enquêtés montrent cependant que cette connaissance n'est pas toujours reliée à une contextualisation spatiale. Les pratiquants affichent leur incapacité de localiser le palais khedaouedj qui est enchevêtré dans le tissus médinal. Le palais des Rais est plus identifiable par sa position excentrée. 
LECTURE DU RAPPORT COGNITIF AU PATRIMOINE

DANS UNE PERSPECTIVE COMMUNICATIONNELLE : ...

Les réseaux sociaux s'imposent pour leur part comme des supports influents et sont assimilés à une nouvelle agora où chaque citoyen, pourrait donner son avis, poster une photo, trouver ou diffuser une information... etc.

\section{Conclusion}

$\mathrm{Au}$ travers de l'analyse des entretiens nous pouvons avancer les résultats suivants.

L'appropriation cognitive du patrimoine bâti se révèle dans une configuration différenciée en fonction du profil des enquêtés et leurs expériences du lieu.

- Elle se construit en faveur d'une intériorisation cognitive par la familiarisation, la pratique ou l'usage d'un espace.

- Ce constat se vérifie dans une résolution optimum avec les résidents qui présentent une maitrise cognitive du site. La transmission jouant à ce niveau le rôle d'un canal de compétence intergénérationnelle.

- Cette compétence sera plus variable avec les travailleurs qui investissent cognitivement des rayons d'espaces supports de leurs pratiques habituelles.

- En dehors de ces configurations, les données révèlent que les compétences cognitives des enquêtés sont insuffisantes et ce à tous les niveaux de leur expression.

- Cette défaillance de la compétence cognitive est reportée par les enquêtés à la déficience ou l'absence de dispositifs informatifs dans l'espace public.

- La médiation s'impose de ce fait comme un facteur opérant sur la construction d'une appropriation cognitive. Elle agira dans une interaction permanente entre le niveau de connaissance des enquêtés et les dispositifs mobilisés à des fins de compréhension et de lisibilité. L'objectif étant de permettre aux enquêtés d'attribuer une valeur à ce avec quoi ils sont en contact.

- La médiation in situ est citée comme la démarche la plus efficiente pour développer une connaissance sur le patrimoine bâti du centre historique. La signalétique émerge en faveur des réponses comme le dispositif le plus opérant à cet objectif.

Le processus d'appropriation cognitive est de fait une construction binaire qui repose sur deux facteurs : 
I. En premier lieu la pratique du site. Cette dernière permet une interrelation avec le lieu qui s'apparente à une réception intime de l'espace par le sujet social. L'appropriation dans ce sens, se définit dans un processus d'accumulation expérientielle et relationnelle avec le lieu.

II. Le deuxième facteur est défini par la médiation qui intervient dans le processus d'appropriation par les compétences qu'elle développe chez le sujet social en termes d'interprétation et de construction de sens. Elle agit sur sa capacité non seulement acquérir les connaissances transmises mais à les raccrocher à sa propre expérience pour produire de la signification.

- Ce capital informatif optimise la maitrise cognitive et spatiale du centre historique

- Dans cette logique, l'appropriation est considérée comme un processus sociocognitif qui est activé ou renforcé par la médiation dans l'objectif de réaliser la maitrise d

\section{BIBLOGRAPHIE}

Blanchet, Alain et Gotman, Anne, 1992 L'enquête et ses méthodes : l'entretien, Nathan Université, Paris.

Caune, Jean, 1999, Pour une éthique de la médiation. Le sens des pratiques culturelles, PUG.

Bourouiba, Rachid, 1983, L'art religieux musulman en Algérie, SNED, Alger.

Darra, Bernard, 2004, "Etudes des Conceptions de la Culture et de la Médiation », in Marie Thonon (Dir.) Médiation Médiateur, L'Harmattan / MEI, Paris, pp. 61-85.

Davallon, Jean, 2004, «La communication en procès », in Médiation et Information (MEI), $\mathrm{n}^{\circ} 19$, pp. 37-58.

Devoulx, A., 1870, "Les édifices religieux de l'ancien Alger », in La Revue Africaine, Edition Bastide, Alger, p. 226.

Djermoune, Nadir \& Oubouzar, Leila, 2011, « De l'orientalisme éclectique à l'abstraction moderne. Une lecture typologique des architectures algéroises des XIXe et XXe siècles », in Bacha Myriam (dir.), Architectures au Maghreb (XIX ${ }^{e}-X X^{e}$ siècles). Réinvention du patrimoine, Presses Universitaires Francois-Rabelais. Collection « Villes et Territoires ».

Feinanguai, Bruno, 2004, «Les modalités d'appropriation territoriale de l'espace urbain », in Rupture Nouvelle série $N^{\circ} 5$, Mutation, défis en Afrique australe, Publication de l'association rupture-Solidarité, pp. 244-255.

Fischer, Gustave-Nicolas, 1992, Psychologie Sociale de l'Environnement, éditeur Privat, Toulouse. 
LECTURE DU RAPPORT COGNITIF AU PATRIMOINE

DANS UNE PERSPECTIVE COMMUNICATIONNELLE : ...

Fourquet-Courbet, Marie-Pierre, 1999, «Un siècle de théories de l'influence : histoire du procès des médias », in Médiation et Information $n^{\prime}{ }^{\circ} 10$, Histoire et Communication, L'Harmattan, Paris, pp. 101-116.

Godard, Olivier, 1990, «Environnement, modes de coordination et systèmes de légitimité : Analyse de la catégorie de patrimoine naturel ", in Revue économique, $\mathrm{n}^{\circ} 41$, mars, pp. 215-241.

Golvin, Lucien, 2003, Palais et demeures d'Alger à la période ottomane, Edition Inas, Alger.

Jacobi, Daniel, Jeanneret, Yves, 2013, « Du panneau à la signalétique : lecture et médiations réciproques dans les musées », in Davallon, Jean, Gottesdiener, Hana, Cultures et musées, hors-série, La Muséologie : 20 ans de recherches, Association Publics et Musées, Université d'Avignon, Actes Sud, Arles, pp. 47-72.

Lamizet, Bernard, 1999, La médiation culturelle, L'Harmattan, Paris.

Mucchielli, Alex, 1996 «Pour des recherches en communication, Communication et organisation» [En ligne], 10 I mis en ligne le 26 mars 2012, consulté le 22 décembre 2019. URL : http://journals.openedition.org/communicationorganisation/187

Paille, Pierre, Mucchielli, Alex, 2008, L'analyse qualitative en sciences humaines et sociales, Armand Colin, Paris.

Passini, Romedi, 1984, Wayfinding in architecture, Van Nostrand Reinhold New York, Toronto.

Passini, R. Shiels, 1987, G. \& Canada. Travaux publics Canada. Services D'architecture et de Génie, "S'orienter dans les édifices publics: une ligne dïrectrice », Travaux publics Canada. Services d'architecture et de génie. Technologie, Ottawa.

Raffestin, Claude, 1980, Pour une Géographie du pouvoir, LITEC, Paris.

Rautenberg, Michel, 2003, La rupture patrimoniale, édition A la croisée, Paris.

Ripoll, Fabrice et Veschambre, Vincent, 2005, «Introduction », in Norois, n 195, pp. 7-15.

Serfaty-Garzon, Perla, 2003, "L'Appropriation », in Segaud, Marion, Brun, Jacques, Driant, Jean-Claude (dir), Dictionnaire critique de l'habitat et du logement, éditions Armand Colin, Paris, pp. 27-30.

Stock, Mathis, 2003, « Pratiques des lieux, modes d'habiter, régimes d'habiter : Pour une analyse trilogique des dimensions spatiales des sociétés humaines », in: Travaux de l'Institut Géographique de Reims, vol. 29-30, n¹15-118, pp. 213-229.

Stock, Mathis, 2007, «Théorie de l'habiter. Questionnements », in Thierry Paquot (éd.), Habiter, le propre de l'humain. Villes, territoire et philosophie. La Découverte, pp. 103-125.

Veschambre, Vincent, 2008, Traces et mémoires urbaines, enjeux sociaux de la patrimonialisation et de la démolition, PUR. 
